\title{
Advanced PON Topologies with Wireless Connectivity
}

\author{
Milos Milosavljevic, Pandelis Kourtessis, Member, IEEE \\ Ali Gliwan, Student Member, IEEE, John M. Senior \\ Optical Networks Group, Science and Technology Research Institute (STRI), University of Hertfordshire \\ College Lane Campus, Hatfield, AL10 9AB, UK \\ Tel: +44(0) 1707285082,e-mail:P.Kourtessis@herts.ac.uk
}

\begin{abstract}
The interoperability of wireless and PON networking solutions is investigated to reduce deployment expenditure by means of centralised network management while providing ubiquitous access connections and mobility. Network modelling in the physical layer of WiMAX channel transmission based on FDM over legacy PONs has demonstrated EVMs below $-30 \mathrm{~dB}$ and error-free multipath transmission. In addition, the development of a dynamic MAC protocol suite has been presented to assign bandwidth between the OLT and ONU BaseStations over a multi-wavelength, splitter-PON topology to demonstrate converged network scalability. This has been achieved by managing data-centric traffic with quality of service in view of diverse multi-user access technologies.
\end{abstract}

Keywords: interoperability, PON, WiMAX, radio over fibre (RoF), dynamic multi wavelength (DMW).

\section{INTRODUCTION}

Although the escalating demand in bandwidth provision at close subscriber proximity could be widely met by optical networking solutions, next generation access networks should also provide end users with great flexibility and mobility at ease of last-mile implementation [1]. To that extent convergence of optical and the rapidly growing by means of deployment and standards wireless networking, e.g. WiMAX and LTE, represents the challenge for ubiquitous broadband multimedia communications. A novel architectural platform demonstrating the transparent transmission of radio signals over standardized passive optical network (PON) topologies has been investigated in that direction [2]. The application of Radio over Fibre (RoF), for enhanced network redundancy and capacity through centralised management, is in the focus by means of accessing each remote Optical Network Unit/BaseStation (ONU/BS) from the optical line terminal (OLT) by frequency division multiplexing (FDM). In addition, for enhanced scalability and to overcome network congestion of single wavelength networks, a dynamic multi-wavelength (DMW) GPON protocol methodology has been designed to support multi-wavelength operation over currently deployed, power-splitting GPON infrastructures, requiring the slightest modifications in network hardware [3]. Consequently, this would result in reduced network upgrade and components costs.

\section{NETWORK ARCHITECTURE}

The network architecture, depicted in Fig. 1, consists of a standard GPON with novel wireless-enabled OLT and ONUs. At the OLT after WiMAX symbols are generated by multiple transmitters they are upconverted to a subcarrier for each wireless-enabled ONU. The FDM technique was used to ensure signal transparency for various radio signal formats and to avoid interference with the GPON spectrum [2]. Each WiMAX transmitter at the OLT is serving a single radio cell at an ONU/BS that is divided into small parts called sectors in order to make the cell more efficient in terms of reduced co-channel interference and increased capacity. The ONU/BSs

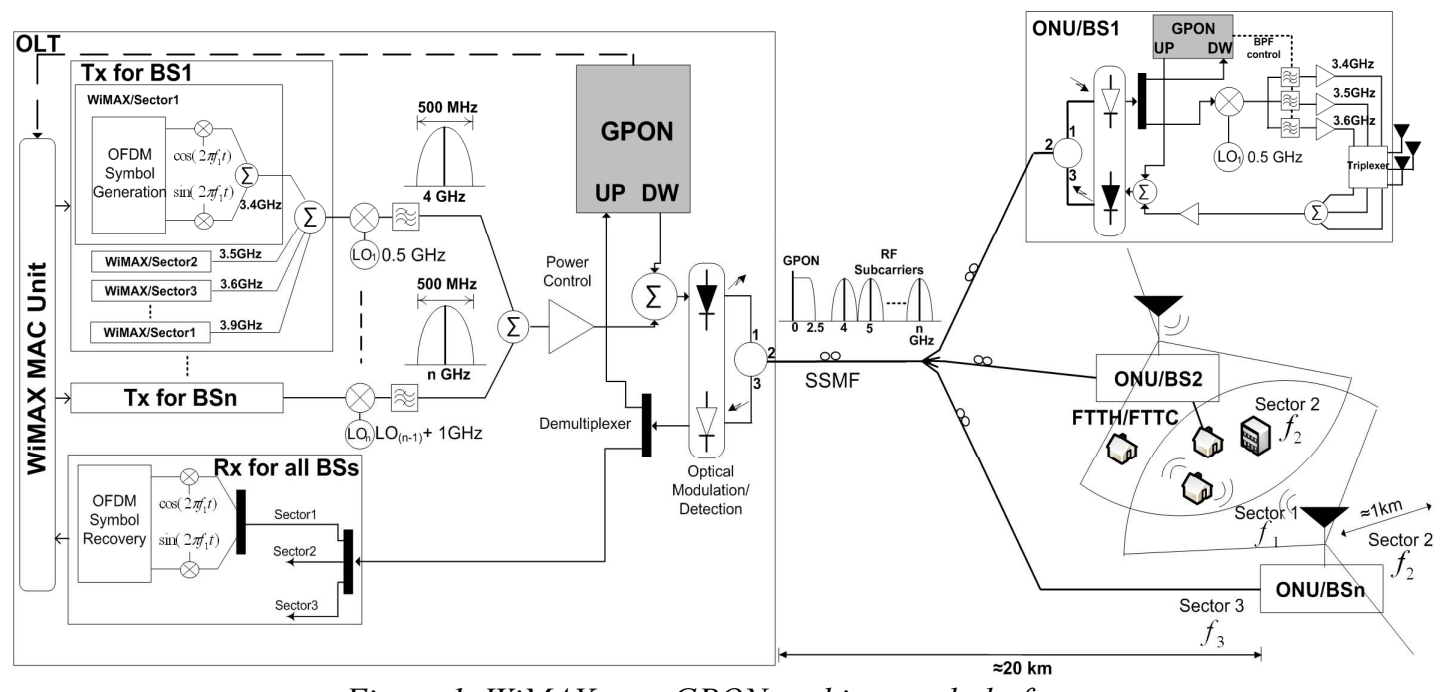

Figure 1. WiMAX over GPON architectural platform. 
are therefore responsible only for downconversion of received channels and for GPON processing.

The key feature of the proposed architecture is creation of overlapping cells, e.g. between Sector 2 and Sector 1 of ONU/BS2 and ONU/BSn respectively in Fig. 1, operating at different frequency channels enabled by centralised processing in the OLT [2]. Therefore, users that are in the overlapping regions can have simultaneous wireless support from multiple ONU/BSs, thus increasing the capacity of the WiMAX network and providing redundancy in case of fibre failure between a distribution node and an ONU due to established alternative routes for signal transmission. Furthermore, the smaller radio cells offered by the application of RoF technique enables much higher spectral efficiency, then in traditional WiMAX deployment, and on-demand bandwidth provisioning by means of additional WiMAX channel for a particular sector from the OLT. Finally, the use of time division multiple access (TDMA) approach in upstream minimises the optical beat interference in the OLT receiver reducing the overall design complexity through a single photoreceiver and WiMAX demodulator.

\subsection{Network performance evaluation}

In order to demonstrate the capability of the architecture to transmit WiMAX channels successfully over combined GPON and radio-cell links a physical layer simulation test-bed was implemented using Virtual Photonic Inc. (VPI) and MATLAB [2]. It has been previously established that non-linear effects of the optical modulators can degrade RoF transmission in terms of error vector magnitudes (EVMs) and bit error rates (BERs) [2]. Fig. 2 displays EVMs for five WiMAX channels as a function of the RF drive power into the Mach-Zehnder modulator (MZM) in the OLT, to indicate if the maximum allowable RF drive power into the MZM complies with the WiMAX transmitter requirements at an ONU/BS. For low input power levels into the MZM the performance is limited by noise, while for high power levels, nonlinear distortions of the MZM increase EVMs. For the 64-QAM WiMAX OFDM downstream transmitters an EVM of $-31 \mathrm{~dB}$ was obtained at distinctive drive powers, matching closely the performance figure of the WiMAX standard [4].

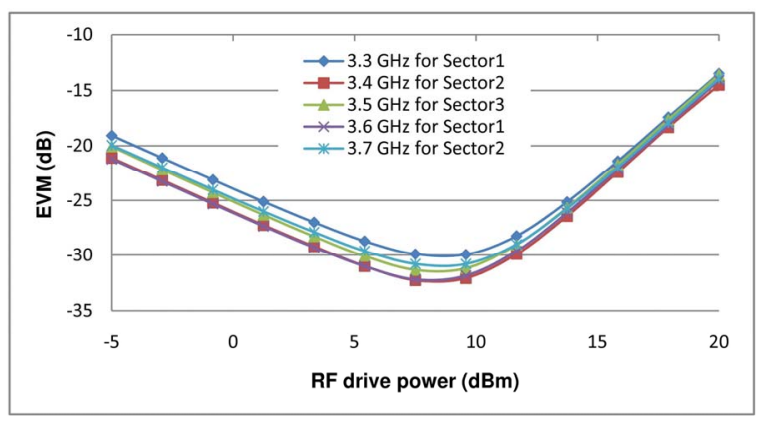

Figure 2. EVM versus RF drive power for downstream WiMAX channels.
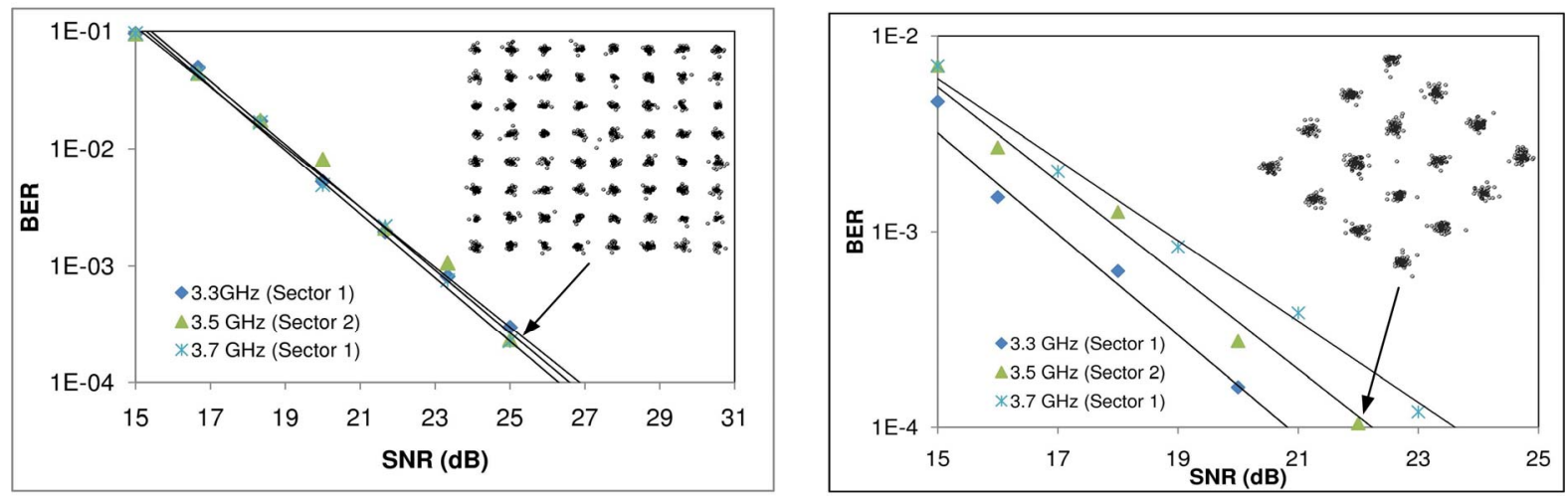

Figure 3. BER versus SNR for 64-QAM (left) and 16-QAM (right) OFDM channels bidirectionally.

To demonstrate signal transmission across GPON and radio links, BER plots for three WiMAX channels bidirectionally are shown in Fig. 3. In downstream the proposed architecture has displayed BER figures of 1E-4, which are considered as error free transmission for IPTV and online gaming services [5], at an SNR requirement of at least $26 \mathrm{~dB}$ with $-30 \mathrm{dBm}$ fixed received power at the APD [6]. The application of wireless channel coding techniques is expected to reduce the recorded worst-case SNRs. Similar results were obtained for the other channels. In addition, this demonstrates $50 \mathrm{Mbit} / \mathrm{s}$ aggregate downstream capacities at transmission links extended to $21 \mathrm{~km}$ as opposed to relatively short distances achieved with standard WiMAX deployment. The wireless channel at $3.7 \mathrm{GHz}$ deployed from the OLT could be used for on-demand bandwidth provisioning or for redundancy in case of fibre failure through overlapping cells. In upstream, for WiMAX channels, comprising 16-QAM OFDM modulation, the BER responses displays power penalty of $3 \mathrm{~dB}$ at $10^{-4}$ between the $3.3 \mathrm{GHz}$ and $3.7 \mathrm{GHz}$ channels due to laser chirp introduced by direct laser modulation (DML). 
The constellation diagrams, shown as insets in Fig. 3, represent the obtained data points where upstream channel DML caused constellation rotation.

\section{EXTENDED WAVELENGTH BAND OVERLAY}

Scalability of the presented topology to larger splits by means of additional ONU/BSs, would result in extended RF spectrum occupancy, exceeding bandwidth specifications of electrical and optical devices and imposing high dispersion penalty. In addition, this would increase polling waiting time for wireless users in upstream due to the TDMA approach. Alternatively the application of extended wavelength band overlay over the PON to assign each ONU a unique P2P logical connection with the OLT provides the undisputable solution for low cost electrical and optical components in the OLT and ONU/BSs with reduced polling time. This will also enable smooth upgrade from the currently deployed TDM- to WDM-PONs with higher splitting ratios and minor modifications in the field infrastructure [7-9]. In hardware, this could be implemented by the addition of an array waveguide grating (AWG) in the OLT, tuneable optical filters and low-cost colourless VCSEL arrays, not limited to Rayleigh backscattering, in ONU/BSs.

To that extent, a DMW GPON protocol is designed to increase upstream capacity and provide support for multi-wavelength operation over the currently deployed GPON infrastructures at the slightest modifications in the frame formats and network hardware. The continuous use of the splitter in the remote node (RN) offers the OLT the ability to switch between upstream wavelengths among ONUs and also share these wavelengths in the time domain [3]. This has been demonstrated by reviewing the upstream and downstream frame format maps and consequently developing a new protocol based on the experience gained from dynamic bandwidth allocation (DBA) algorithms for single-wavelength operation [3]. Both gate and report packets of GPON, used to establish communication between the OLT and ONUs, were adjusted in order to support DMW operation. Out of the twelve bits in the Flags-field, six unused reserved bits were utilised where four bits are assigned to express ONU's operating wavelength for proceeding cycles and two bits to specify the packet type.

\subsection{DMW protocol mechanism}

The DMW protocol at a first stage represents the extension of the previously implemented dynamic minimum bandwidth (DMB) protocol [10] to support multi-wavelength operation. The DMW protocol, in comparison to $\mathrm{DMB}$, employs four service levels and extra upstream wavelengths to specify finer ONU service requirements, presented in Fig. 4, as well as to provide an additional level of flexibility in assigning each ONU maximum allowed bandwidth by means of multiple wavelengths.

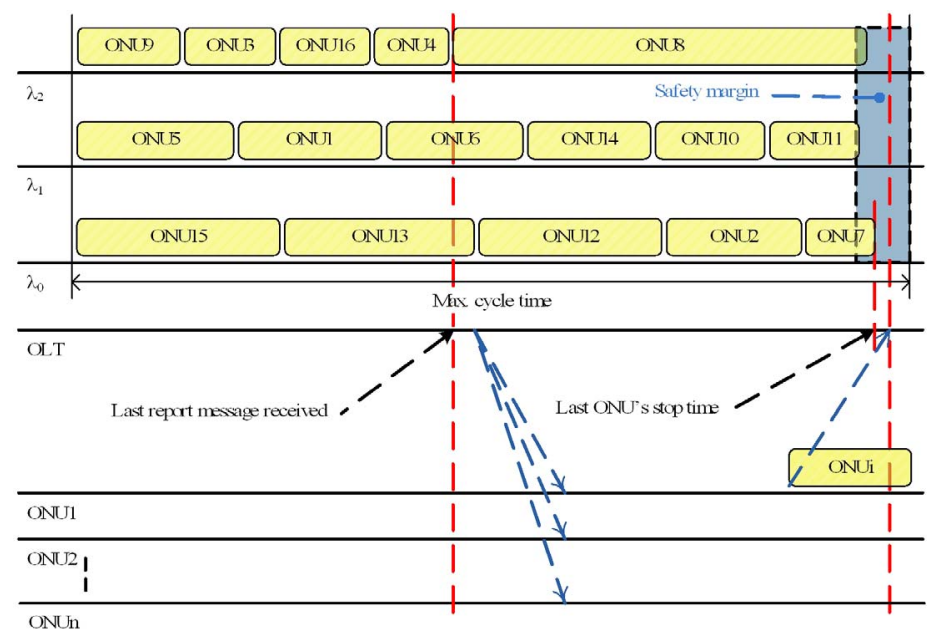

Figure 4. DMW-GPON nrotocol nrincinles.

As demonstrated in Fig. 4, the OLT will send request to each ONU to confirm their supported wavelengths each time an ONU registers on the network. After all reports are received from the network ONUs, the upstream bandwidth assignment will be performed in three stages. In the first stage, the OLT allows for a safety margin and then allocates each ONU bandwidth according to the total network capacity remaining after having subtracted the margin. The maximum allocated bandwidth for $\mathrm{ONU}_{\mathrm{i}}$ is assigned according to the DMB protocol by considering the new total network capacity [3]. To minimise the mean packet delay due to idle bandwidth slots formed in traditional single-wavelength PONs, the second stage was introduced. This is achieved by assembling ONU traffic in the OLT according to their assigned bandwidth from low to high, as shown in Fig. 4, keeping the ONU with the highest transmission time last for improved network throughput. In the third stage, the OLT commences assignment of the ONU time-slots to different wavelengths sequentially starting from $\lambda_{\text {up } 0}$ up to $\lambda_{\text {up2 }}$ (in case of three wavelengths). Therefore, the OLT guarantees the last ONU time-slot in $\lambda_{\text {upi }}$ fits within the 
safety margin. This approach produces a shorter polling cycle while reducing ONU upstream packets waitingtime for the proceeding cycles resulting in an evident increase in network utilization [3].

Consequently, while in single-wavelength DMB-GPON algorithms with 32 ONUs and four SLAs the minimum-bandwidth for the highest-service-level-ONUs is limited to $35 \mathrm{Mbit} / \mathrm{s}$, as derived from Fig. 5, the DMW-GPON protocol provides each ONU with more than a $100 \mathrm{Mbit} / \mathrm{s}$ as a minimum guaranteed bandwidth to its equivalent higher-service-level-ONU. Significantly, it exhibits lower mean packet delay and enhanced network utilisation, as demonstrated in Fig. 5, in comparison to single-wavelength operation.
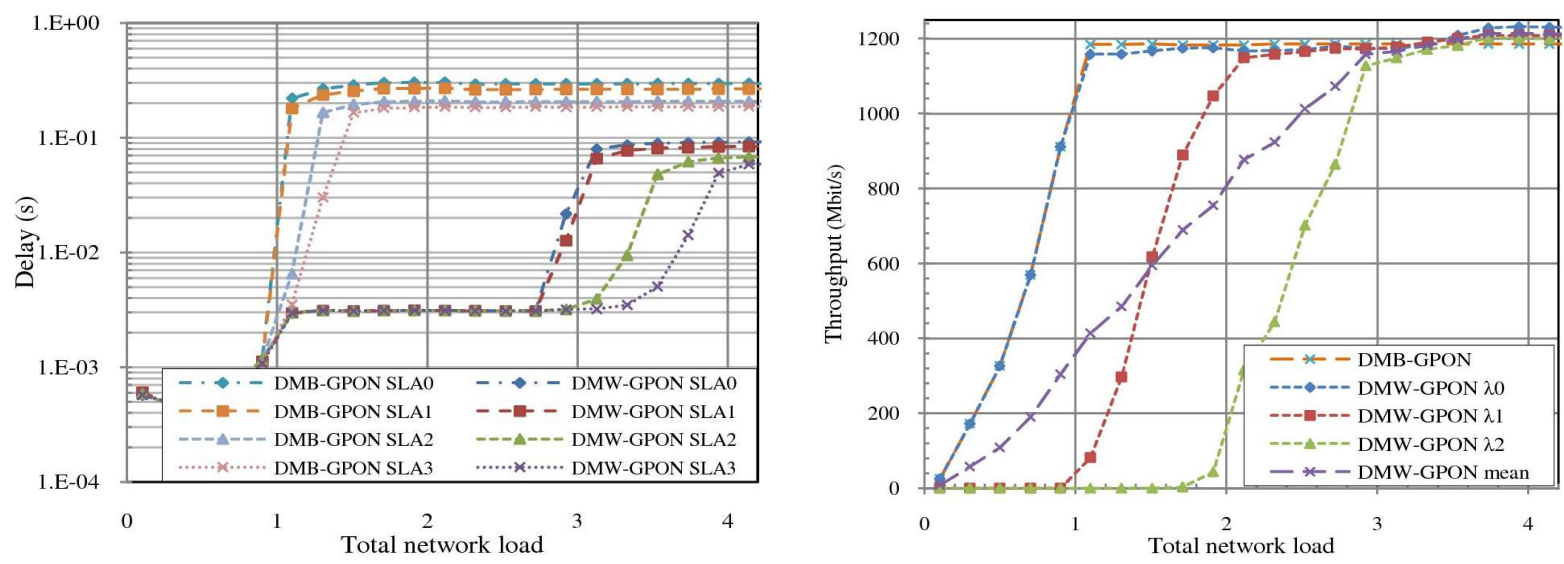

Figure 5. Mean packet delay (left) and throughput (right) versus network load for DMB-and DMW-PON

\section{CONCLUSIONS}

The innovative architectural platform described in this paper exploits the merits of standard GPON and WiMAX technologies to capture the best attributes of each, seeking to facilitate ubiquitous connection together with the low deployment cost and mobility. The maximum EVMs of $-30 \mathrm{~dB}$ in downstream was obtained as required by typical WiMAX transceivers while error free transmission of standard WiMAX rates have been demonstrated bidirectionally for extended link lengths up to $21 \mathrm{~km}$. The centrally controlled ONU/BSs, in comparison with distributed control in a typical WiMAX deployment, has allowed for the formation of overlapping cells which have enabled GPON redundancy in case of fibre failure between a distribution point and an ONU and enhanced WiMAX capacity. In addition, the DMW protocol methodology and the corresponding enhancements in the GPON upstream format map have been presented to accommodate multi-wavelength operation over the network optical domain by means of smoothly upgrading the existing single-wavelength GPON infrastructure. This upgrade has been achieved by utilising additional bits in the transmission frame fields to define the operating wavelength and packet type transfer for each ONU. The performance merits of multi-wavelength operation, providing a minimum $100 \mathrm{Mbit} / \mathrm{s}$ transfer rates per ONU have been demonstrated through OPNET simulations.

\section{REFERENCES}

[1] G. Shen, R. S. Tucker, and C.-J. Chae: Fixed mobile convergence architectures for broadband access: Integration of EPON and WiMAX, IEEE Communications Mag., vol. 45, pp. 44-50, Aug. 2007.

[2] M. Milosavljevic, Y. Shachaf, P. Kourtessis, and J. M. Senior: Interoperability of GPON and WiMAX for network capacity enhancement and resilience, J. Optical Netw., vol. 8, pp. 285-294, Apr. 2008.

[3] A. Gliwan, C. Chang, Y. Shachaf, P. Kourtessis, and J. M. Senior: Upstream format map enhancements for multi-wavelength GPONs, in Proc. NOC, Krems, Austria, July, 2008, pp. 75-82.

[4] "IEEE standard for local and metropolitan area networks, Part 16: Air interface for fixed broadband wireless access systems," IEEE Std 802.16-2004 (Revision of IEEE Std 802.16-2001), pp. 0_1-857, (2004).

[5] K. N. Skalman, H. E. Sandstrom, and M. Gidlund: Techno-economical study for open horizontal IPTV in the access and home networks - from a Swedish perspective, in Proc. NOC, Krems, Austria, July 2008, 218-229.

[6] G.984.2: Gigabit-capable passive optical networks (G-PON): Physical Media Dependent (PMD) layer specification (ITU-T, 2003).

[7] R. Davey, J. Kani, F. Bourgart, and K. McCammon: Options for future optical access networks, IEEE Communications Mag., vol. 44, pp. 50-56, Oct. 2006.

[8] L. G. Kazovsky, W. T. Shaw, D. Gutierrex, N. Cheng, and S. W. Wong: Next-generation optical access networks, J. Lightwave Technol., vol. 25, pp. 3428-3442, Nov. 2007.

[9] G.984.5: Enhancement band for gigabit capable optical access networks (ITU-T, 2007).

[10] C. H. Chang, P. Kourtessis, and J. M. Senior: GPON service level agreement based dynamic bandwidth assignment protocol, Electronics Lett., vol. 42, pp. 1173-1174, Sept. 2006. 